\title{
Preparation of cycloaliphatic epoxy hybrids with non-conventional amine-curing agents
}

\author{
María González González • Juan Carlos Cabanelas • \\ Javier Pozuelo $\cdot$ Juan Baselga
}

\begin{abstract}
Cycloaliphatic epoxy resin has been successfully thermally cured using a polyamino siloxane oligomer as hardener. The curing reaction was followed by infrared spectroscopy in the near range, and thermal transitions were measured by dynamic mechanical thermal analysis. The use of accelerants and a plasticizer (dodecylphenol), and different curing schedules were explored. Cured materials showed high $T g$ values (around $125164{ }^{\circ} \mathrm{C}$ ), and the analysis of laser scanning confocal microscopy images showed that they are homogeneous in the microscale.
\end{abstract}

Keywords Thermal curing · Polysiloxane .

Cycloaliphatic epoxy $\cdot$ Glass transition

\section{Introduction}

Epoxy resins are widely employed as adhesives, coatings, and encapsulates as well as in polymer matrix composites. Its application in several industries is often related with its low viscosity, making the uncured material suitable for shaping complex components [1]. There are several families of epoxy resins differing in the thermal and mechanical class. Within the resins for medium- or low-curing temperatures, the most conventional ones are glycidyl ethers of bisphenol A (DGEBA) and cycloaliphatic epoxies.

Due to its very low viscosity, good insulating properties even at high temperatures, good chemical resistance and low dielectric constant, cycloaliphatic epoxy resins have

M. G. González · J. C. Cabanelas · J. Pozuelo · J. Baselga ( $₫)$ Dpto. Ciencia e Ingeniería de Materiales e Ingeniería Química, University Carlos III of Madrid (IAAB), Av. Universidad 30, 28911 Leganés, Madrid, Spain

e mail: jbaselga@ing.uc3m.es long been used as insulating materials for electrical devices (transformers) and for encapsulation of electronic components [1]. The absence of UV chromophores makes this family of epoxies also suitable for UV curing and outdoor applications, although in the case of alicyclic resins, its inherent brittleness and hydrolytic degradation at high temperature and humidity limit their use [2].

Differences in chemical structure make cycloaliphatic epoxies reactivity completely different from the reactivity of common DGEBA epoxy resins. The aromatic ether linkage in DGEBA has a strong electron-withdrawing effect making the oxirane group highly reactive towards nucleophilic compounds (like amines), whilst the cyclohexyl group in cycloaliphatic epoxies does not have this strong effect, making this kind of compounds reactive towards Lewis acids like anhydrides instead of amines [3]. Additionally, a protecting effect of axial and equatorial protons of the cyclohexyl ring against nucleophilic attack has been proposed as an explanation of the characteristic low reactivity of the oxirane ring in these alicyclic epoxies [4]. This is the main reason why the best performance and the highest crosslinking degree for DGEBA-based resins is achieved when cured via an addition mechanism with diamines (either aliphatic or aromatic), whilst cycloaliphatic epoxies are commonly cured with anhydrides [2, 5 7] or homopolymeriszed via a cationic mechanism [8 13$]$. For the later, the preferred superacid initiators are generated in situ either thermally [8, 9] or photochemically [11, 12], although the curing mechanism is not fully understood.

3,4-Epoxycyclohexyl-3'4'-epoxycyclohexane carboxilate (ECC) is an interesting low viscosity commercial alicyclic diepoxide. This resin has recently been used in the preparation of radiation-cured nanocomposites filled with montmorillonite [14], bohemite [18] and $\mathrm{LaF}_{3}$ [15]. Other nanoparticles such as nanosilica have been studied 
focusing on their toughening effect [20] or as a means to reduce the shrinking on cure [21, 22], although alternative ways for reducing shrinking by copolymerization with lactones have been also reported [23]. The kinetics of both, the UV cationic curing [16] and the anhydride thermal curing [19] have been studied for the neat resin as well as for modified systems with dendritic-toughening additives [17] and with surface-modified $\mathrm{SiO}_{2}$ particles [24].

In view of the alleged lack of reactivity against amines, no literature has been found on the curing of this diepoxide with aliphatic amines. In this work, we explore the use of a non-conventional organo-inorganic polyamine as curing agent for the ECC resin. This hardener has been previously used for DGEBA epoxy resins [25 30], showing very good reactivity at moderate temperatures but yielding a heterogeneous morphology on the cured samples as revealed by dynamic light scattering and dynamic mechanical thermal analysis [30]. The origin of this heterogeneity has two main possible sources: the initial mutual insolubility of both the components and the microphase separation during cure. The former is related with the competition between chemical reaction, which occurs at the interface of the siloxane-rich domains, and diffusion of the nascent chains throughout the system. The latter, must be related with non favourable interactions between the nascent chains and some or both of the initial components.

ECC is a low polarity resin. If it is used instead of DGEBA, then it can be envisaged as having a better miscibility with the polyaminosiloxane and, as a consequence, a more homogeneous microstructure is expected. As it will be shown, we report that this curing agent is able to react with this resin using similar conditions as the ones used for anhydrides, although the aid of a plasticizer is necessary to decrease the curing time. Dynamic mechanical thermal analysis results are compatible with the presence of inhomogeneities in the network structure at the macromolecular scale, but a detailed analysis of confocal images will show that no inhomogeneities are present at the micro scale.

\section{Experimental}

ECC was provided by Daicel Chemical Industries (Japan) with an epoxy equivalent weight of $132 \mathrm{~g} \mathrm{eq}^{-1}$. $m$-xylylenediamine was purchased from Aldrich. Poly(3aminopropylmethyl-siloxane) (PAMS) was synthesised by hydrolysis and condensation according to a method published elsewhere [25].

Amine equivalent weight was $58.5 \mathrm{~g} \mathrm{eq}^{-1}$ active hydrogen, and average number molecular weight determined by vapour pressure osmometry was $M_{n}=910 \mathrm{~g} \mathrm{~mol}^{-1}$. Chemical formulae of the compounds are given in Table 1. Dodecylphenol was purchased from Aldrich; accelerant for
Table 1 Chemical structures of the epoxy compound and polyamine hardener

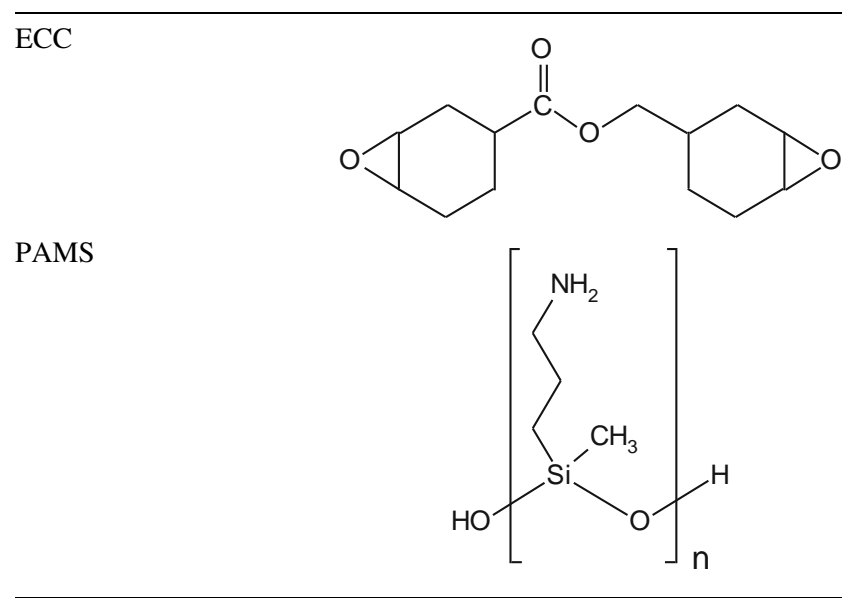

the curing reaction (Gairesa 1265) was an aqueous/organic solution (30\% water) of inorganic salts.

For confocal microscopy, PAMS was reacted with Rhodamine B sulphonyl chloride (Lissamine ${ }^{\circledR}$, Molecular Probes). Reaction was carried out under nitrogen atmosphere at $60{ }^{\circ} \mathrm{C}$ for $4 \mathrm{~h}$. Final concentration of the chromophore in PAMS was $10^{-4}$ mol kg-1 $(1$ chromophore per 6700 polymer chains). Samples were prepared mixing stoichiometric amounts of the epoxy component and PAMS and stirring for $1.5 \mathrm{~min}$ at room temperature.

For infrared spectroscopy in the near range (FTnIR, Perkin Elmer GX2000) studies, an appropriate amount of the mixture was placed between two glass slides separated by a Teflon spacer with a thickness not higher than $0.64 \mathrm{~mm}$. The sandwich was placed inside a Specac thermostatic sample chamber at the desired curing temperature. Five scans were collected per spectrum at $8 \mathrm{~cm}^{-1}$ resolution in the range $75004000 \mathrm{~cm}^{-1}$.

DMTA measurements (Rheometrics Scientific, Mk III) were performed in the single cantilever bending mode between 30 and $250{ }^{\circ} \mathrm{C}$ at $2{ }^{\circ} \mathrm{C} \mathrm{min}{ }^{-1}$ at $1 \mathrm{~Hz}$. $\mathrm{Tg}$ values were calculated as the temperature at the maximum of tan $\delta$. Samples were prepared by moulding a previously degassed resin mixture in a $20 \times 10 \times 2 \mathrm{~mm}^{3}$ steel mould. Curing schedule was $120{ }^{\circ} \mathrm{C}, 0.5 \mathrm{~h}$ followed by a 2-h step at $155^{\circ} \mathrm{C}$ and a final postcure at $190{ }^{\circ} \mathrm{C}$ for $1 \mathrm{~h}$.

Laser scanning confocal microscopy images (LSCM, Zeiss LSCM-5 Pascal) were taken of samples modified with Rhodamine. Excitation was carried out with a $25-\mathrm{mW}$ Ar laser at $488 \mathrm{~nm}$ and an Ultrafluar $40 \times / 0.6$ immersion objective (scanned area $230 \times 230 \mu \mathrm{m}$ ) was used. In all the cases, images were taken at $100 \mu \mathrm{m}$ from the surface. Samples for LSCM observation were prepared between two cover slides separated by a 1-mm silicone spacer, and cured in an oven at the desired temperature. Images were taken after quenching at room temperature. 


\section{Results}

Curing process

Curing of ECC with PAMS was followed by infrared spectroscopy in the near range since ECC absorptions in the mid range overlap with PAMS absorptions making the quantitative analysis difficult. In Fig. 1, the initial FTIR spectrum of ECC-curing system in the near range is presented. It is worthy to note that the oxirane combination band (bending + stretching) usually located at around $4530 \mathrm{~cm}^{-1}$ for common epoxies cannot be observed in the NIR spectrum of the ECC blend. Therefore, the epoxy conversion cannot be measured by FTIR. Instead, we have measured the primary amine conversion $(\beta)$ following the combination band (bending + stretching) at $4938 \mathrm{~cm}^{-1}$. For long reaction times, when primary amines are exhausted, progress of the reaction can be qualitatively followed from the primary and secondary amine combination band at $6530 \mathrm{~cm}^{-1}[31,32]$.

In Fig. 2 the primary amine conversion of ECC/PAMS system at $120{ }^{\circ} \mathrm{C}$ is presented and compared with the curing of ECC with a common carbon-based diamine: $m$-xylylenediamine (mXDA) [33 35]. It can be observed that the siloxane hardener shows enhanced reactivity when compared with mXDA: the global kinetic constant for PAMS is three times larger than for mXDA. Nevertheless, reaction time at which primary amine is exhausted is very long compared with DGEBA under similar conditions, suggesting the need of an accelerator. In Fig. 2 it can be observed that if a small quantity of a commercial accelerator (1265, Gairesa) is added, then maximum $\beta$ is considerably shortened from 90 to $15 \mathrm{~min}$ at $120^{\circ} \mathrm{C}$. The global kinetic constant in this case is tenfold than for pure PAMS and 30-fold than m-XDA.

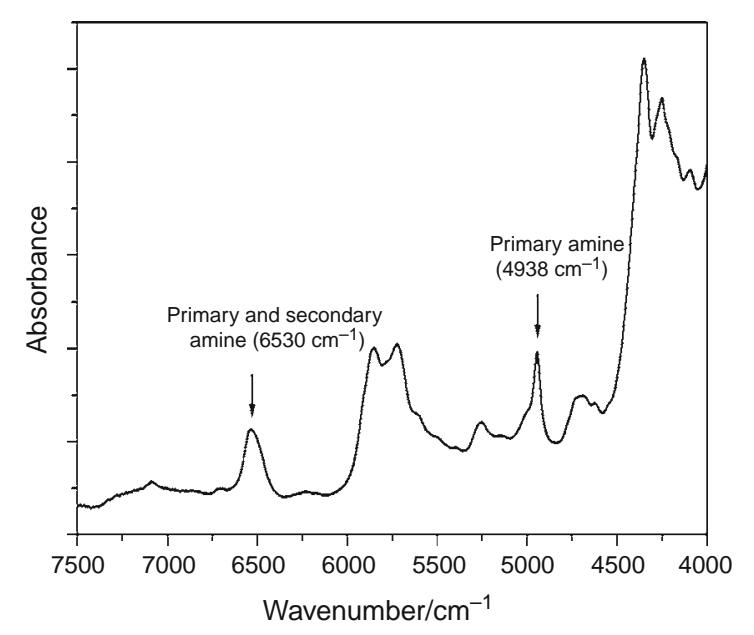

Fig. 1 FTnIR spectrum of ECC/PAMS stoichiometric initial mixture

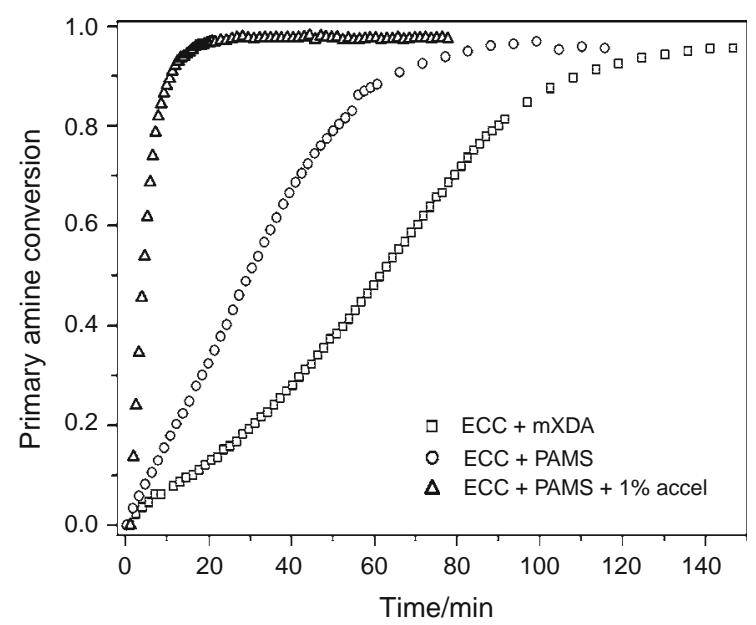

Fig. 2 Primary amine conversion versus time for ECC/PAMS with and without accelerant and ECC/mXDA at $120^{\circ} \mathrm{C}$

The relative change of the primary and secondary combination band at $6530 \mathrm{~cm}^{-1}$ is presented in Fig. 3. It becomes evident (Fig. 3a) that the curing rate slows down shortly after $\beta$ reaches its maximum value, remaining low even if temperature is increased to $150{ }^{\circ} \mathrm{C}$. As a consequence, the glass transition temperature of ECC/PAMS system cured $5 \mathrm{~h}$ at $120^{\circ} \mathrm{C}$ obtained by DMTA is only $50{ }^{\circ} \mathrm{C}$, and the resulting material is a fragile solid. This result indicates that the reactivity of the secondary amine towards the oxirane group of ECC is very low below $120{ }^{\circ} \mathrm{C}$, and remains low even at $190{ }^{\circ} \mathrm{C}$, leading to a long curing cycle. Since it is not possible to cure at higher temperatures due to the low boiling point of the epoxy resin $\left(185^{\circ} \mathrm{C}\right)$, the use of a plasticizer and a non-isothermal temperature programme to increase conversion degree was explored. It has been found that a suitable combination of accelerator, plasticizer and temperature steps to design a processing route which yields high conversions at moderate curing times (Fig. 3b): 1\% of Gairesa-1265 accelerant, 20\% dodecylphenol plasticizer (PH), $30 \mathrm{~min}$ at $120{ }^{\circ} \mathrm{C}$ followed by two temperature steps at $150{ }^{\circ} \mathrm{C}(2 \mathrm{~h})$ and at $190{ }^{\circ} \mathrm{C}$ $(1 \mathrm{~h})$. Under these curing conditions, a very high conversion is reached in $3.5 \mathrm{~h}$, a relatively short time in comparison with curing protocols used for anhydride hardeners [2].

It should be noted that dodecylphenol bears hydroxyl groups that may catalyse the addition reaction. No specific study of this effect has been done.

The absence of amide formation during the curing reaction at high temperatures was checked by FTIR both in the near and the mid range. The Amide I and Amide II bands located at 1650 and $1550 \mathrm{~cm}^{-1}$, respectively, [36] were absent in infrared spectra in the medium range of cured samples, as well as the amide combination band $(v \mathrm{NH}$ (asymmetric) + stretching Amide II) at $50255128 \mathrm{~cm}^{-1}$ in the near range [37]. It was also observed that the ester 


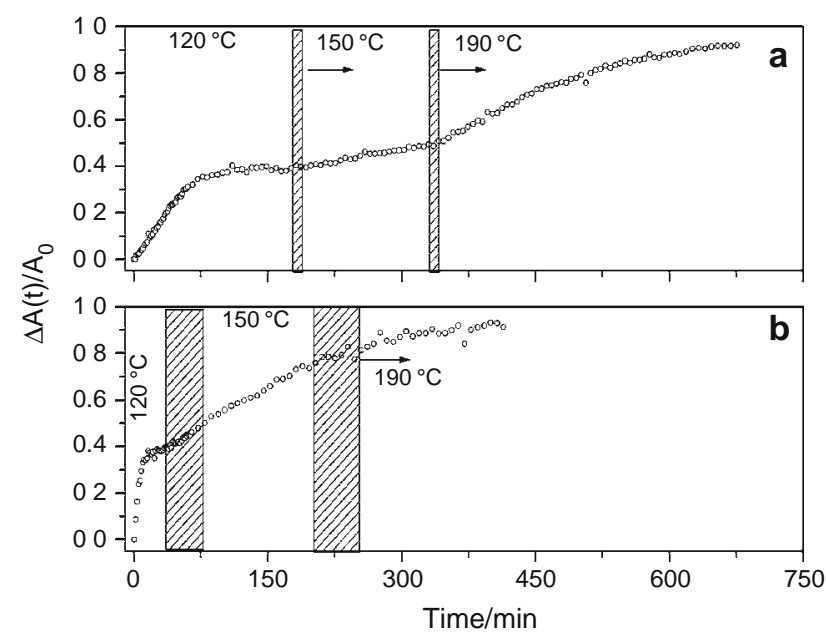

Fig. 3 Evolution of the primary and secondary combination band at $6530 \mathrm{~cm}^{-1}$ versus reaction time. Effect of nonisothermal curing protocol for ECC/PAMS (a) and influence of 1\% accelerator and $20 \%$ dodecylphenol (b)

stretching $\mathrm{C}=\mathrm{O}$ band located at $1735 \mathrm{~cm}^{-1}$ remains unaltered in the cured samples.

Thermal transitions

Dynamic mechanical thermal analysis was performed on different ECC/PAMS cured samples and the results are shown in Figs. 4 and 5 and in Table 2. The relaxation behaviour of ECC cured with PAMS is quite complex. As previously mentioned, when curing ECC up to full primary amine conversion at $120^{\circ} \mathrm{C}, \tan \delta$ shows a narrow band peaking at $50{ }^{\circ} \mathrm{C}$ (Fig. 4). If the complete curing schedule is applied until total amine conversion is almost reached, this relaxation assigned to the $\mathrm{Tg}$, shifts to $164{ }^{\circ} \mathrm{C}$

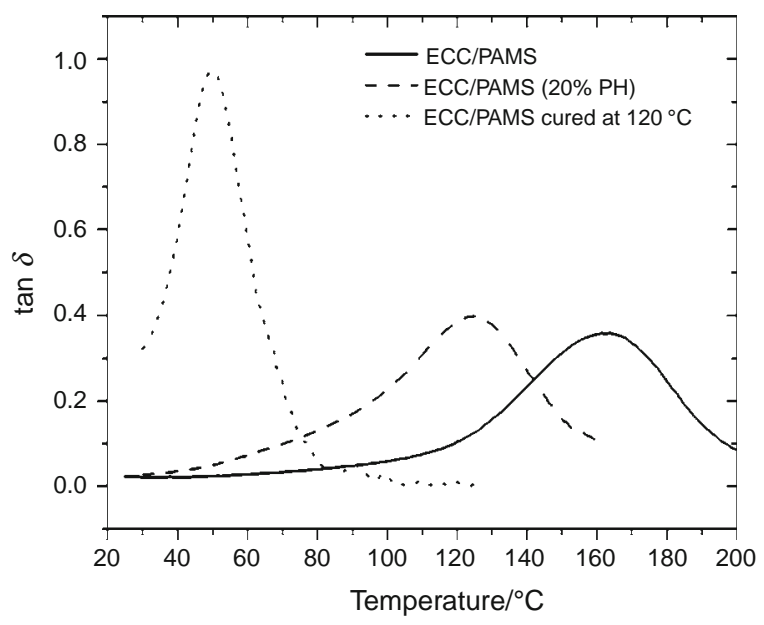

Fig. 4 Tan $\delta$ as a function of temperature for ECC/PAMS cured samples: at $120^{\circ} \mathrm{C}$ (dots) with accelerant and plasticizer (dashes) and with accelerant only (continuous line)

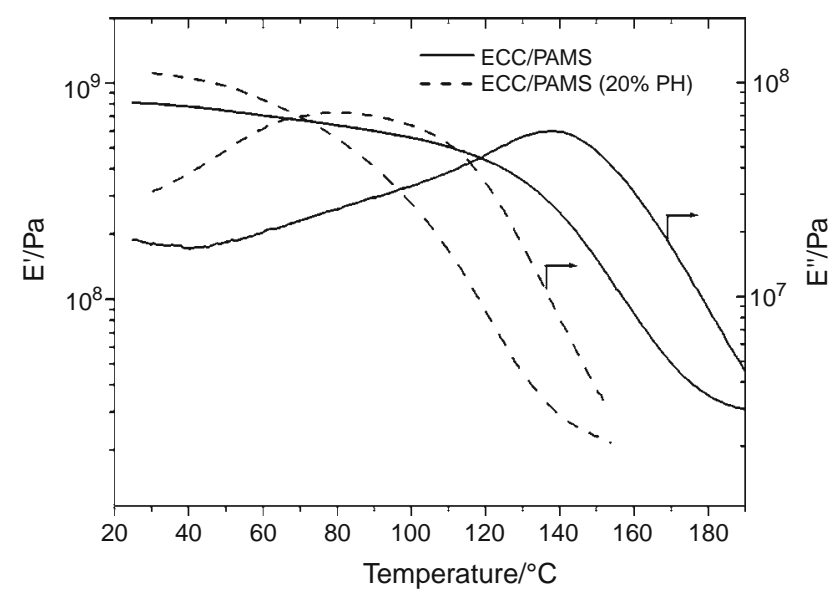

Fig. 5 Storage $\left(E^{\prime}\right)$ and loss $\left(E^{\prime \prime}\right)$ moduli as a function of temperature at $1 \mathrm{~Hz}$ for neat ECC/PAMS system (continuous line) and with $20 \%$ dodecylphenol as plasticizer (dash)

Table 2 Glass transition temperature $(T g)$, maximum $\tan \delta\left(\tan \delta_{\max }\right)$ and halfwidth of $\tan \delta(w)$ for ECC/PAMS systems

\begin{tabular}{lcll}
\hline System & $T g /{ }^{\circ} \mathrm{C}$ & $\tan \delta_{\max }$ & $w /{ }^{\circ} \mathrm{C}$ \\
\hline ECC/PAMS cured at $120{ }^{\circ} \mathrm{C}$ & 50 & 0.97 & 23 \\
ECC/PAMS & 164 & 0.36 & 53 \\
ECC/PAMS (20\%PH) & 125 & 0.40 & 50 \\
\hline
\end{tabular}

indicating that crosslinking density was far less the maximum when the sample was cured at $120{ }^{\circ} \mathrm{C}$, in agreement with conversion data. The $T g$ of the fully cured material is appreciably higher than that previously reported for DGEBA cured with aliphatic amines, including PAMS [29], probably due to the absence of flexible ether bonds in ECC. It is worthy to note that the $T g$ is in the same temperature range as reported for this resin cured with anhydrides [2] and slightly lower than that reported for UV curing under extremely high UV intensity [27].

An additional interesting feature is the increase of the halfwidth of $\tan \delta$ band ( $w$, see Table 2) from 23 to $53{ }^{\circ} \mathrm{C}$. Cured homogeneous DGEBA resins typically show halfwidths around $30{ }^{\circ} \mathrm{C}$ [38], and values reported for ECC resins cured with anhydrides are around $34{ }^{\circ} \mathrm{C}$ [2]. If DGEBA is cured at high temperature with the hardener used in this work, then the halfwidth increases to $43{ }^{\circ} \mathrm{C}$ [30]. Therefore, the large half-width observed in ECC/ PAMS suggests the presence of additional relaxation mechanisms associated to the presence of the polysiloxane. The analysis of the shape of the $\alpha$ relaxation can provide useful information about the structure of the crosslinked network [39]. The height of the transition is related to the extent of cure and decreases as the distance between crosslinks shortens. Tan $\delta$ height is clearly higher for ECC/ PAMS partially cured at $120{ }^{\circ} \mathrm{C}$ because of the low 
crosslinking density of the network, but it is significantly low for the fully cured material compared with common epoxy-amine systems, probably due to the high functionality of the PAMS oligomer which induces a higher crosslinking degree. The height can be related to the network homogeneity in terms of changes in the crosslinking density especially for samples with very high $T g$ [40], although the broadening can be also related to chemical clustering or microphase segregation.

In Fig. $4, \tan \delta$ variation for the sample containing 20\% dodecylphenol is also presented. The $T g$ of the final material is lower than that the observed for the neat ECC/ PAMS system suggesting that dodecylphenol acts as an effective plasticizer for these systems. Halfwidth shows no significant changes in comparison with the system without plasticizer (Table 2).

Storage and loss moduli as a function of temperature are presented in Fig. 5. The essential features of this figure are: (i) an antiplastification effect with an associated increase of the room temperature modulus of about $30 \%$, which is quite common for many thermosetting systems [38], (ii) maximum of loss modulus for the plasticized system broadens and peaks at about $80{ }^{\circ} \mathrm{C}, 30^{\circ} \mathrm{C}$ below the observed peak in $\tan \delta$ (Fig. 4), (iii) the rubbery modulus is lower for the plasticized system as expected for swollen networks.

According to the well-known expression, $M_{\mathrm{C}}=\rho R T / E$, crosslinking density could be estimated from the rubbery modulus for the neat system case. For the plasticized system, a correction for swelling must be applied to calculate $M_{\mathrm{C}}$ : for swollen networks $E=\rho R T \phi^{-1 / 3} / M_{C, S}$, where $\phi$ is the polymer volume fraction. Since density at high temperature is not known, the only magnitude that can be calculated is the ratio between molecular weights between crosslinks for the neat and plasticized systems assuming constant density. This ratio is $M_{\mathrm{C}} / M_{\mathrm{C}, \mathrm{S}} \approx 0.1$, indicating that the low rubbery modulus is due to a lower crosslinking degree. The lower concentration of reactive groups (dilution effect) may be a plausible explanation.

It is worth noting that loss modulus peaks are very broad for both systems, which is consistent with the large bandwidths found in $\tan \delta$. As mentioned above, there are two possible origins of this broadening: microphase separation during cure and inhomogeneous distribution of both components due to the initial mutual insolubility. In previous work with DGEBA resins, it was found that reaction between the epoxy and the polyaminosiloxane takes place at the interface between these two phases [27 30]. During curing, the epoxy component diffuses through the PAMS domains making them to grow. At this stage, diffusion and reaction compete, and if temperature is not very high, then compositional gradients are irreversibly formed in the cured material. The inhomogeneous composition distribution has been characterised by SEM [27] and laser scanning confocal microscopy, being the last one particularly suitable for studying these systems since the domains size and compositional gradients are in the range of tens of microns.

\section{Image analysis}

The presence of composition inhomogeneities in ECC/ PAMS systems in the range of several microns was characterized by fluorescence laser scanning confocal microscopy using the Rhodamine B-labelled hardener as shown in the Experimental part. Images of the ECC/PAMS systems without (a) and with dodecylphenol (b) are presented in Fig. 6 at two curing stages: initially and after applying the curing schedule. For the neat system, the initial image shows bright domains, which correspond to PAMS rich regions, embedded in the ECC liquid matrix reflecting the initial insolubility of both components. As curing proceeds, the heterogeneous structure disappears yielding an almost homogeneous film. Compared with the previously reported DGEBA/PAMS system [29], the behaviour of ECC/PAMS is quite similar, except for the fact that homogeneity at the micro scale is experimentally achieved in the early stages of the curing and also the homogeneity at the last stages of the cure process is higher.

The initial morphology completely changes when dodecylphenol is added (Fig. 6b). This image probably corresponds to some transient structure between initial mixture and the equilibrium state. However, in any case, as expected for non-ionic surfactants [41], dodecylphenol reduces the interfacial tension between both the components, decreasing

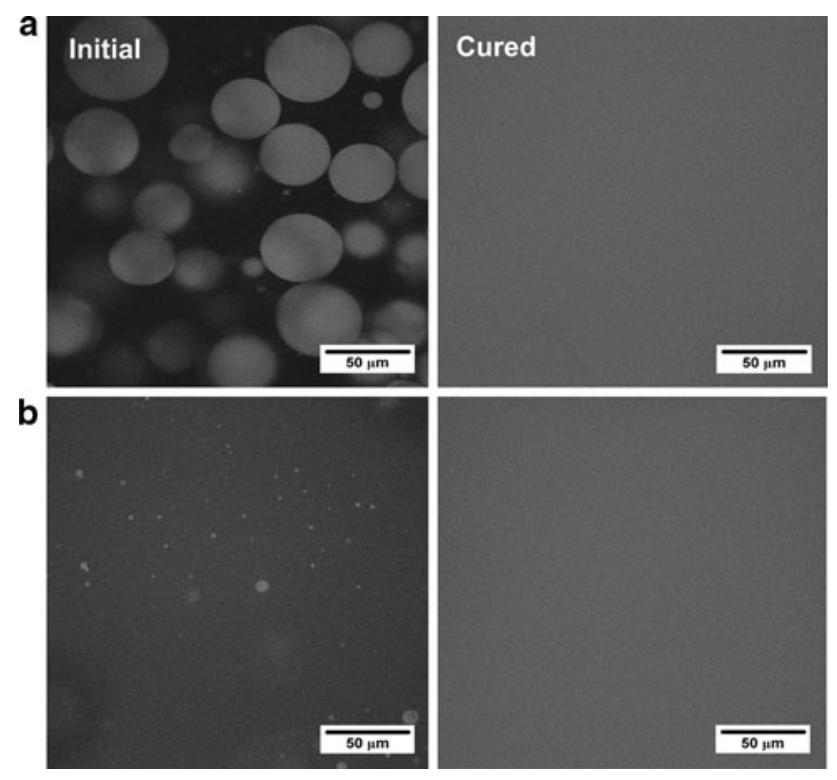

Fig. 6 Confocal images of the ECC/PAMS systems immediately after mixing (initial) and after curing; a without dodecylphenol, b with dodecylphenol and accelerant. Image size was $250 \times 250 \mu \mathrm{m}$ 
the size of the PAMS droplets from $40 \mu \mathrm{m}$ to about $3 \mu \mathrm{m}$ as an average, and enhancing mutual solubilisation. The final material appears to be also homogeneous at the micron scale as in the previous case.

\section{Conclusions}

Compared to other common amines, such as mXDA, polyaminosiloxane hardener (PAMS) shows an enhanced reactivity against cycloaliphatic epoxy resins. The curing process can be improved with commercial accelerants and plasticizers. A curing schedule was developed yielding materials with high $T g$ and shortened curing times; both, $T g$ and curing time, are similar to the ones found when using anhydrides as curing agents. Confocal images reveal that the material is homogeneous, indicating that the initial dual-phase morphology is completely lost during the quiescent curing process and is not transferred to the cured material at the micron scale. Analysis of the loss moduli and $\tan \delta$ reveals the existence of additional relaxation mechanisms for this system, irrespectively of the addition of plasticizer. According to the previous work, two possible sources of heterogeneities may be at the origin of the loss moduli broadening: microphase separation during cure and inhomogeneous distribution of both components due to the initial mutual insolubility. However, these two sources must operate at a lower scale.

Acknowledgements The authors would like to express their grati tude to the Dinater (MAT2007 63722) and Interfases (S 0505/ MAT0227) projects for financial support.

\section{References}

1. May CA. Epoxy resins, chemistry and technology. New York: Marcel Dekker; 1988.

2. Wang Z, Xie M, Zhao Y, Yu Y, Fang S. Synthesis and properties of novel liquid ester free reworkable cycloaliphatic diepoxides for electronic packaging application. Polymer. 2003;44:923 9.

3. Mark HF. Encyclopaedia of polymer science and technology. New Jersey: Wiley; 2004. vol 9.

4. Soucek MD, Abu Shanab OL, Anderson CD, Wu S. Kinetic modeling of the crosslinking reaction of cycloaliphatic epoxides with carboxyl functionalized acrylic resins: Hammett treatment of cycloaliphatic epoxides. Macrom Chem Phys. 1998;199: 103542.

5. Barabanova AI, Shevnin PL, Pryakhina TA, Bychko KA, Kaz antseva VV, Zavin BG, Vygodskii YS, Askadskii AA, Philippova OE, Khokhlov AR. Nanocomposites based on epoxy resin and silicon dioxide particles. Polym Sci. 2008;50:808 19.

6. Tao Z, Yang S, Chen J, Fan L. Synthesis and characterization of imide ring and siloxane containing cycloaliphatic epoxy resins. Eur Polym J. 2007;43:1470 9.

7. Chen JS, Ober CK, Poliks MD. Characterization of thermally reworkable thermosets: materials for environmentally friendly processing and reuse. Polymer. 2002;43:131 9.
8. Crivello JV. Ring opening polymerization. München: Hanser; 1995. p. 15796.

9. Yagci Y, Reetz I. Externally stimulated initiator systems for cationic polymerization. Progr Polym Sci. 1998;23:1485 538.

10. Hartwig A, Koschek K, Luhring A, Schorsch O. Cationic poly merization of a cycloaliphatic diepoxide with latent initiators in the presence of structurally different diols. Polymer. 2003;44: 28538.

11. Crivello JV, Fan M. Novel platinum containing initiators for ring opening polymerizations. J Polym Sci Polym Chem. 1991; 29:1853 63 .

12. Wang F, Neckers DC. Photopolymerization of epoxides with platinum(II) bis(acetylacetonato)/silane catalysts. Macromole cules. 2001;34:6202 5 .

13. Crivello JV, Liu S. Photoinitiated cationic polymerization of epoxy alcohol monomers. J Polym Sci Polym Chem. 2000;38: 389401.

14. Malucelli G, Bongiovanni R, Sangermano M, Ronchetti S, Priola A. Preparation and characterization of UV cured epoxy nano composites based on o montmorillonite modified with maleinized liquid polybutadienes. Polymer. 2007;48:7000 7.

15. Sangermano M, Roppolo I, Shan G, Andrews MP. Siloxane additive as modifier in cationic UV curable coatings. Progr Org Coat. 2006;57:44 9.

16. Voytekunas VY, Ng FL, Abadie MJM. Kinetics study of the UV initiated cationic polymerization of cycloaliphatic diepoxide resins. Eur Polym J. 2008;44:3640 9.

17. Corcione CE, Malucelli G, Frigione M, Maffezzoli A. UV cur able epoxy systems containing hyperbranched polymers: kinetics investigation by photo DSC and real time FT IR experiments. Polym Test. 2009;28:157 64.

18. Corcione CE, Frigione M, Maffezzoli A, Malucelli G. Photo DSC and real time FT IR kinetic study of a UV curable epoxy resin containing o boehmites. Eur Polym J. 2008;44:2010 23.

19. Harsch M, Krger Kocsis J, Holst M. Influence of fillers and additives on the cure kinetics of an epoxy/anhydride resin. Eur Polym J. 2007;43:1168 78.

20. Zhang X, Xu W, Xia X, Zhang Z, Yu R. Toughening of cyclo aliphatic epoxy resin by nanosize silicon dioxide. Mat Lett. 2006;60:3319 23.

21. Chen MH, Chen CR, Hsu SH, Sun SP, Su WF. Low shrinkage light curable nanocomposite for dental restorative material. J Prosth Dent. 2006;22:138 45.

22. Chen MH, Chen CR, Hsu SH, Sun SP, Su WF. Low shrinkage light curable nanocomposite for dental restorative material. Dent Mat. 2006;22:138 45.

23. Fernandez X, Ramis X, Salla JM. Cationic copolymerization of cycloaliphatic epoxy resin with an spirobislactone with lantha num triflate as initiator: kinetics of the curing process. Thermo chim Act. 2005;438:144 54.

24. Hartwig A, Sebald M, Kleemeier M. Cross linking of cationi cally polymerised epoxides by nanoparticles. Polymer. 2005;46: 202939.

25. Cabanelas JC, Serrano B, González Benito J, Bravo J, Baselga J. Morphology of epoxy/polyorganosiloxane reactive blends. Macrom Rap Com. 2001;22:694 9.

26. Prolongo SG, Cabanelas JC, Baselga J. Reactive compatibiliza tion of epoxy/polyorganosiloxane blends. Macrom Symp. 2003; 198:283 93.

27. Cabanelas JC, Prolongo SG, Serrano B, Bravo J, Baselga J. Water absorption in polyaminosiloxane epoxy thermosetting polymers. J Mat Proc Tech. 2003;143:311 5.

28. Cabanelas JC, Serrano B, Baselga J. Development of cocontin uous morphologies in initially heterogeneous thermosets blended with poly(methyl methacrylate). Macromolecules. 2005;38: 96170. 
29. Cabanelas JC, Serrano B, González MG, Baselga J. Confocal microscopy study of phase morphology evolution in epoxy/ polysiloxane thermosets. Polymer. 2005;46:6633 9.

30. Gonzalez MG, Kadlec P, Stepanek P, Strachota A, Matejka L. Crosslinking of epoxy polysiloxane system by reactive blending. Polymer. 2004;45:5533 41.

31. Mijovic J, Andjelic S. A study of reaction kinetics by near infrared spectroscopy. 1 comprehensive analysis of a model epoxy/amine system. Macromolecules. 1995;28:2787 96.

32. Kradjel C, Lee KA. NIR analysis of polymers. In: Handbook of near infrared analysis. Burns DA, Ciurczak EW, Eds. Boca Raton: CRC Press; 2008. p. 55631.

33. Villanueva M, Martín Iglesias JA, Rodríguez Anón JA, Proupín Castineiras. Thermal study of an epoxy system DGEBA (n 0 )/ mXDA modified with POSS. J Therm Anal Cal. 2009;96:575 82.

34. Maria Villanueva, Fraga I, Rodríguez Anón JA, Proupín Casti neiras J, Martín JL. Study of the influence of water absorption on different epoxy diamine systems by DSC. J Therm Anal Cal. 2007;87:205 9 .

35. Lisardo Núnez Regueira, Villanueva M, Fraga I, Gracia Fernán dez CA, Gómez Barreiro S. Influence of the thermal degradation on the dielectric properties of a thermoset. J Therm Anal Cal. 2006;86:235 41 .

36. Li L, Seino H, Yonetake K, Ueda M. Synthesis and character ization of ordered poly(amide ester)s from Isophthaloyl Chloride and 4 (2 Aminoethyl)phenol. Macromolecules. 1999;32:3851 8.

37. Chop YS, Kim J, Park J, Yu J A, Yoon C J. Near infrared spectroscopic studies of the hydrogen bonding between thioace tamide and $\mathrm{N}, \mathrm{N}$ disubstituted benzamide derivatives in $\mathrm{CCl} 4$. Spectrochim Act A. 1996;52:1779 83.

38. Tcharkhtchi A, Lucas AS, Trotignon JP, Verdu J. Viscoelastic properties of epoxy networks in the glass transition region. Polymer. 1998;39:1233 5.

39. Gerard JF, Galy J, Pascault JP, Cukierman S, Halary JL. Visco elastic response of model epoxy networks in the glass transition region. Polym Eng Sci. 1991;31:615 21.

40. Pascault JP, Sautereau H, Verdu J, Williams RJJ. Thermosetting Polymers. New York: Marcel Dekker; 2002. p. 334.

41. Weinheimer RM, Varineau PT. Nonionic surfactants: Organic chemistry. Surfactant Science Series. New York: Marcel Dekker; 1998. 